\title{
Cycloaddition of substituted spiroepoxycyclohexa-2,4-dienones: synthesis and X-ray crystal structure of the adducts ${ }^{\dagger}$
}

\author{
Vishwakarma Singh, ${ }^{\mathrm{a}, *}$ Dilip K. Tosh, ${ }^{\mathrm{a}}$ Vinayak V. Kane, ${ }^{\mathrm{b}, *}$ Ludger Ernst ${ }^{\mathrm{b}}$ and Peter G. Jones ${ }^{\mathrm{b}}$ \\ ${ }^{a}$ Department of Chemistry, Indian Institute of Technology, Bombay, Mumbai 400 076, India \\ ${ }^{\mathrm{b}}$ Department of Chemistry, TU Braunschweig, Hagenring 30, D-38106 Braunschweig, Germany
}

Abstract-The syntheses of the tricyclic ketoepoxides $\mathbf{1}$ and $\mathbf{2}$ from the readily available aromatic precursors $\mathbf{5}$ and $\mathbf{6}$ via cycloaddition of the spiroepoxycyclohexa-2,4-dienones $\mathbf{3}$ and $\mathbf{4}$ are described. The crystal structures of $\mathbf{1}$ and $\mathbf{2}$ were determined.

There has been a recent renewed interest in the chemistry of cyclohexa-2,4-dienones ${ }^{1,2}$ and masked $o$-benzoquinones. ${ }^{3}$ In particular, $\pi^{4 s}+\pi^{2 s}$ cycloaddition of these systems provides an efficient route to a variety of bicyclo[2.2.2]octenones that undergo a variety of reactions in the ground and excited states, leading to complex polycyclic systems which have served as precursors for diverse natural products. ${ }^{1-3}$ In continuation of our studies in this area, ${ }^{2}$ we required tetracyclic structures 1 and 2 containing a vinyl bromide and an alkene or alkyne tether attached to the oxirane ring. Our aim was to generate vinyl radicals by treating bromo compounds 1 and 2 with $\mathrm{Bu}_{3} \mathrm{SnH} / \mathrm{AIBN}$ and to probe for the intramolecular cyclization of these radicals by reac- tion with the allyl or propargyl multiple bonds. This did not occur. Further, it was of interest to study the compatibility of the bromo substituent and the allyl or propargyl group in the same molecule with the reaction conditions of the $\mathrm{NaIO}_{4}$ oxidation described below. We wish to report here a facile synthesis of tetracyclic compounds 1 and $\mathbf{2}$ from readily available aromatic precursors 5 and $\mathbf{6}$ via their oxidation to the spiroepoxycyclohexa-2,4-dienones $\mathbf{3}, \mathbf{4}$ followed by in situ trapping with cyclopentadiene (Scheme 1).

For the synthesis of compounds $\mathbf{1}$ and $\mathbf{2}$, the aromatic precursors 5 and $\mathbf{6}$ were readily prepared from 5-bromosalicylaldehyde 7 by addition of allyl bromide 4 and

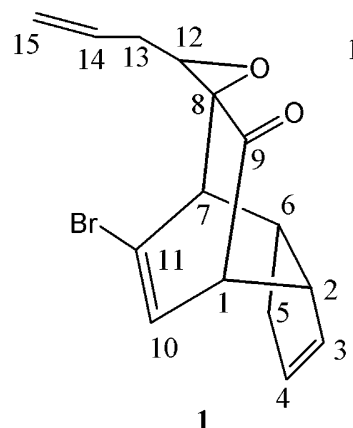

1

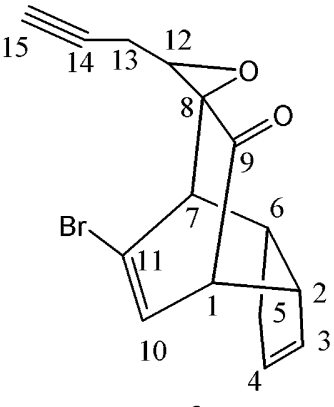

2<smiles>[R]C1OC12C=C(Br)C=CC2=O</smiles>

$3 \mathrm{R}=$ allyl

$4 \mathrm{R}=$ propargyl<smiles>[R]C(O)c1cc(Br)ccc1O</smiles>

$5 \mathrm{R}=$ allyl

$6 \mathrm{R}=$ propargyl

Scheme 1.

Keywords: tetracyclic vinyl bromides with alkene or alkyne tether; 2D NMR; X-ray crystallography. 


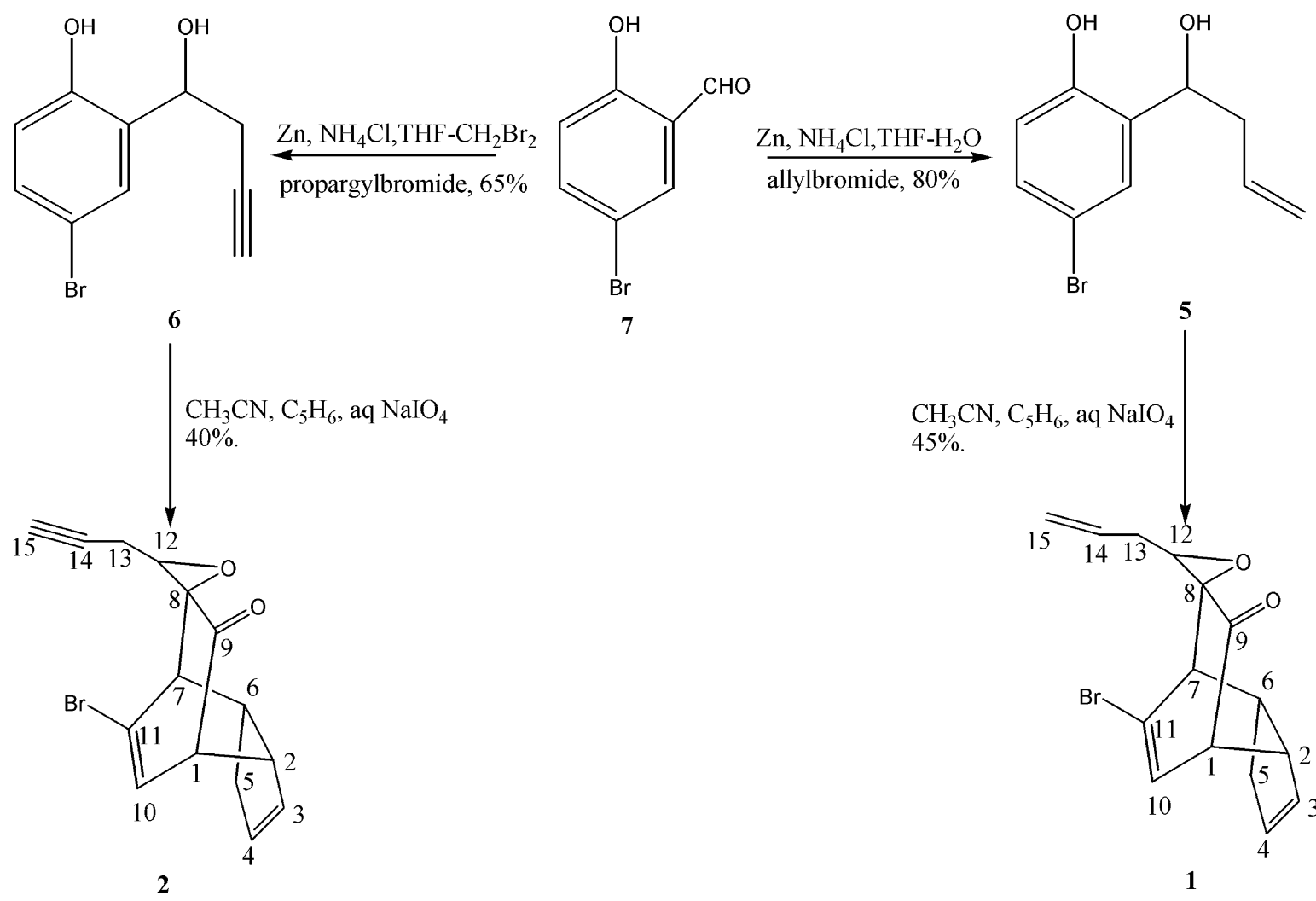

Scheme 2.

propargyl bromide, ${ }^{5}$ respectively, in the presence of $\mathrm{Zn}-\mathrm{NH}_{4} \mathrm{Cl}$ (Scheme 2). Precursors 5 and 6 were oxidized with $\mathrm{NaIO}_{4}$ in the presence of freshly cracked cyclopentadiene following a procedure developed in our laboratory. ${ }^{2 a, 6}$ Chromatography of these products on silica gel gave the adducts $\mathbf{1}$ and $\mathbf{2}$ as crystalline solids in 45 and $40 \%$ yield, respectively. Although the spectral data of these adducts ${ }^{7}$ (full ${ }^{1} \mathrm{H}$ and ${ }^{13} \mathrm{C}$ NMR assignments by $2 \mathrm{D}$ techniques, Table 1) clearly suggested their gross structure, their configurations, especially the orientation of the allyl and propargyl chains and the spirocentre in the oxirane ring were not easily discernible. Therefore, X-ray crystal structures of these adducts were determined (Figs. 1 and 2) that confirmed their stereochemical formulation. ${ }^{8}$

It may be mentioned that, although both adducts are formed as a result of regio- and stereoselective cycloaddition wherein the spiroepoxycyclohexa-2,4-dienones generated in situ behave as the $4 \pi$ partners and cyclopentadiene as the dienophile $2 \pi$ partner, the reaction could, in principle, give a number of products due to various possible modes of addition. ${ }^{9}$

In summary, the synthesis of the new tetracyclic compounds $\mathbf{1}$ and $\mathbf{2}$ from simple and readily available aromatic compounds via in situ generated spiroepoxycyclohexa-2,4-dienones followed by cycloaddition with cyclopentadiene, is described. The structure and stereochemistry of the adducts were established by X-ray single crystal analysis. This cycloaddition represents a precise example of generation of molecular complexity
Table 1. NMR data of compounds 1 and $2\left[\mathrm{CDCl}_{3} / \mathrm{TMS}\right.$; $\left.\delta\left(\mathrm{CDCl}_{3}=77.0\right)\right]$

\begin{tabular}{|c|c|c|c|c|}
\hline \multicolumn{2}{|c|}{ Compound 1} & \multirow[t]{2}{*}{ Position } & \multicolumn{2}{|c|}{ Compound 2} \\
\hline$\delta_{\mathrm{C}}{ }^{\mathrm{a}}$ & $\delta_{\mathrm{H}}^{\mathrm{b}}$ & & $\delta_{\mathrm{C}}$ & $\delta_{\mathrm{H}}^{\mathrm{c}}$ \\
\hline $54.3 \mathrm{~d}$ & $3.44 \mathrm{dd}$ & 1 & $54.2 \mathrm{~d}$ & $3.46 \mathrm{dd}$ \\
\hline $49.7 \mathrm{~d}$ & $3.38 \mathrm{~m}$ & 2 & $49.5 \mathrm{~d}$ & $3.37 \mathrm{dm}$ \\
\hline $134.2 \mathrm{~d}$ & $5.81 \mathrm{dq}$ & 3 & $134.2 \mathrm{~d}$ & $5.81 \mathrm{dq}$ \\
\hline $129.1 \mathrm{~d}$ & $5.45 \mathrm{dq}$ & 4 & $129.0 \mathrm{~d}$ & $5.45 \mathrm{dq}$ \\
\hline $37.6 \mathrm{t}$ & $\begin{array}{l}2.35(\alpha) \mathrm{dtt} \\
2.71(\beta) \mathrm{ddq}\end{array}$ & 5 & $37.6 \mathrm{t}$ & $\begin{array}{l}2.35(\alpha) \mathrm{dddt} \\
2.71(\beta) \mathrm{ddtd}\end{array}$ \\
\hline $37.2 \mathrm{~d}$ & 3.10 dddd & 6 & $37.1 \mathrm{~d}$ & 3.09 dddd \\
\hline $50.7 \mathrm{~d}$ & $2.97 \mathrm{t}$ & 7 & $50.5 \mathrm{~d}$ & $2.95 \mathrm{t}$ \\
\hline $61.8 \mathrm{~s}$ & & 8 & $61.6 \mathrm{~s}$ & \\
\hline $204.0 \mathrm{~s}$ & & 9 & $203.0 \mathrm{~s}$ & \\
\hline $127.6 \mathrm{~d}$ & $6.25 \mathrm{dd}$ & 10 & $127.6 \mathrm{~d}$ & $6.26 \mathrm{dd}$ \\
\hline $120.9 \mathrm{~s}$ & & 11 & $120.6 \mathrm{~s}$ & \\
\hline $62.4 \mathrm{~d}$ & $3.37 \mathrm{t}$ & 12 & $60.8 \mathrm{~d}$ & $3.52 \mathrm{t}$ \\
\hline $33.3 \mathrm{t}$ & $2.47 \mathrm{tt}$ & 13 & $19.6 \mathrm{t}$ & $\begin{array}{l}2.59 \text { (a) ddd } \\
2.68 \text { (b) ddd }\end{array}$ \\
\hline $132.2 \mathrm{~d}$ & $5.89 \mathrm{ddt}$ & 14 & $77.9 \mathrm{~s}$ & \\
\hline $118.4 \mathrm{t}$ & $\begin{array}{l}5.17(E) \mathrm{dq} \\
5.24(Z) \mathrm{dq}\end{array}$ & 15 & $71.5 \mathrm{~d}$ & $2.11 \mathrm{t}$ \\
\hline
\end{tabular}

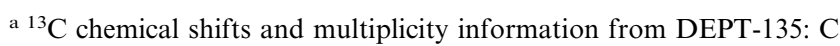
(s), $\mathrm{CH}(\mathrm{d}), \mathrm{CH}_{2}(\mathrm{t})$.

b $J_{1,2} 2.7, J_{1,10} 6.9, J_{2,3} 2.2, J_{2,4} 2.3, J_{2,5 \alpha(?)} 4.0, J_{2,5 \beta(?)} 2.1, J_{2,6} 9.0, J_{3,4}$ $5.6, J_{3,5 \alpha} 2.2, J_{3,5 \beta} 2.2, J_{4,5 \alpha} 2.3, J_{4,5 \beta} 2.3, J_{5 \alpha, 5 \beta} 17.6, J_{5 \alpha, 6} 3.9, J_{5 \beta, 6}$ $9.9, J_{6,7} 3.5, J_{7,10} 2.4, J_{12,13} 6.2, J_{13,14} 6.6, J_{13,15 E} 1.5, J_{13,15 \mathrm{Z}} 1.2$, $J_{14,15 E} 10.2, J_{14,15 \mathrm{z}} 17.2, J_{15 E, 15 Z} 1.4 \mathrm{~Hz}$

${ }^{\mathrm{c}} J_{1,2} 2.6, J_{1,10} 6.9, J_{2,3} 2.2, J_{2,4} 2.3, J_{2,5 \alpha(?)} 3.9, J_{2,6} 8.9, J_{3,4} 5.7, J_{3,5 \alpha}$ $2.2, J_{3,5 \beta} 2.2, J_{4,5 \alpha} 2.3, J_{4,5 \beta} 2.3, J_{5 \alpha, 5 \beta} 17.6, J_{5 \alpha, 6} 4.2, J_{5 \beta, 6} 10.1, J_{6,7}$ $3.2, J_{7,10} 2.5, J_{12,13 \mathrm{a}} 6.0, J_{12,13 \mathrm{~b}} 5.9, J_{13 \mathrm{a}, 13 \mathrm{~b}} 17.5, J_{13 \mathrm{a}, 15} 2.8, J_{13 \mathrm{~b}, 15}$ $2.8 \mathrm{~Hz}$. 


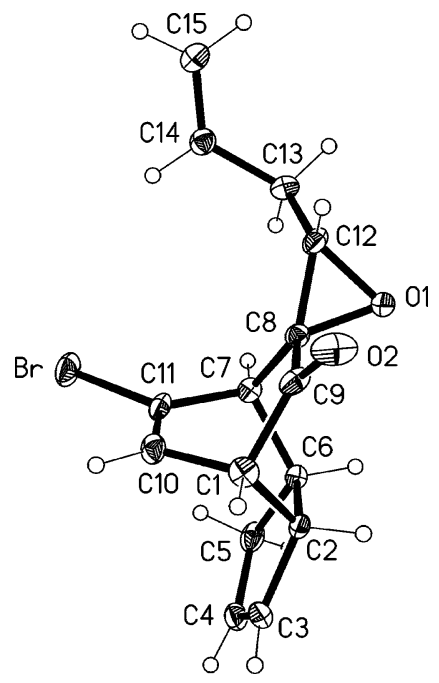

Figure 1. ORTEP diagram for compound 1.

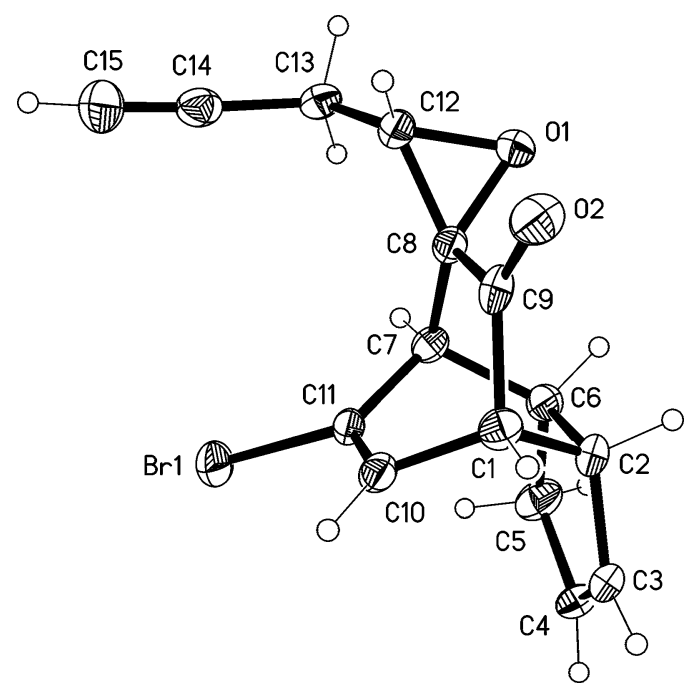

Figure 2. ORTEP diagram for compound 2.

from simple precursors, which is an important aspect of synthetic design. ${ }^{10}$

\section{Acknowledgements}

We thank RSIC, IIT Bombay for spectral data. One of us (D.K.T.) is thankful to CSIR for a fellowship. Continued financial support from CSIR New Delhi is gratefully acknowledged.

\section{References}

1. (a) Yates, P.; Bhamare, N. K.; Granger, T.; Macas, T. S. Can. J. Chem. 1993, 71, 995; (b) Bonnarme, V.; Bachmann, C.; Cousson, A.; Mondon, M.; Gesson, J.-P.
Tetrahedron 1999, 55, 433; (c) Drutu, I.; Njardarson, J. T.; Wood, J. L. Org. Lett. 2002, 4, 493.

2. (a) Singh, V. Acc. Chem. Res. 1999, 32, 324; (b) Singh, V.; Samanta, B.; Kane, V. V. Tetrahedron 2000, 56, 7785; (c) Singh, V.; Alam, S. Q. J. Chem. Soc., Chem. Commun. 1999, 2519.

3. (a) Carlini, R.; Higgs, K.; Rodrigo, R.; Taylor, N. J. Chem. Soc., Chem. Commun. 1998, 65; (b) Chen, Y.-K.; Peddiniti, R. K.; Liao, C.-C. J. Chem. Soc., Chem. Commun. 2001, 1340; (c) Quideau, S.; Pouysegu, L.; Oxoby, M.; Looney, M. A. Tetrahedron 2001, 57, 319.

4. Petrier, C.; Einhorn, J.; Luche, J. L. Tetrahedron Lett. 1985, 26, 1449.

5. Yanagisawa, A.; Habaue, S.; Yamamoto, H. Tetrahedron 1980, 48, 1969.

6. (a) Adler, E.; Brasen, S.; Miyake, H. Acta Chem. Scand. 1971, 25, 2055; (b) Becker, H.-D.; Bremholt, T.; Adler, E. Tetrahedron Lett. 1972, 13, 4205; (c) Adler, E.; Holmberg, K. Acta Chem. Scand. 1974, 28B, 465; (d) Singh, V.; Prathap, S.; Porinchu, M. J. Org. Chem. 1998, 63, 4011.

7. Data for 1: $\mathrm{mp} 63-65^{\circ} \mathrm{C}$. IR $(\mathrm{KBr}) v_{\max }: 1725 \mathrm{~cm}^{-1}$. Found C, 58.43; $\mathrm{H}, 4.89 \% ; \mathrm{C}_{15} \mathrm{H}_{15} \mathrm{BrO}_{2}$ requires $\mathrm{C}$, 58.82; $\mathrm{H}, 4.90 \%$. Data for 2: $\mathrm{mp} 60-62^{\circ} \mathrm{C}$. IR(KBr) $v_{\max }: 1739$ $\mathrm{cm}^{-1}$. Found C, 59.09; $\mathrm{H}, 4.36 \% ; \mathrm{C}_{15} \mathrm{H}_{13} \mathrm{BrO}_{2}$ requires $\mathrm{C}$, $59.01 ; \mathrm{H}, 4.20 \%$.

8. Crystal structure determinations. Compound 1: Crystal data: orthorhombic, space group Pbca, $a=10.3437(8)$, $b=15.5376(16), c=16.4484(16) \AA, U=2643.5 \AA^{3}, Z=8$, $T=-100^{\circ} \mathrm{C}$. Data collection: A crystal ca. $0.45 \times 0.4 \times 0.12$ $\mathrm{mm}$ was used to record 3260 intensities on a Siemens P4 diffractometer (Mo $\mathrm{K} \alpha$ radiation, $2 \theta_{\max } 50^{\circ}$ ). Structure refinement: The structure was refined anisotropically on $F^{2}$ (program SHELXL-97, G.M. Sheldrick, University of Göttingen) to $w R_{2} 0.059, R_{1} 0.032$ for 164 parameters and 2315 unique reflections. The hydrogens were refined using a riding model or rigid methyl groups. Compound 2: Crystal data: Triclinic, space group $P \overline{1}, a=8.0530(10)$, $b=8.1604(10), c=19.766(2) \AA, \alpha=86.716(8), \beta=85.357$ (10), $\gamma=82.766(10)^{\circ}, U=1282.9 \AA^{3}, Z=4, T=-100^{\circ} \mathrm{C}$. Data collection: A crystal ca. $0.4 \times 0.4 \times 0.15 \mathrm{~mm}$ was used to record 5169 intensities as above. Structure refinement: The structure was refined as above to $w R_{2} 0.057, R_{1} 0.028$ for 333 parameters and 4482 unique reflections.

Complete crystallographic data (excluding structure factors) have been deposited at the Cambridge Crystallographic Data Centre under the numbers CCDC-184661 (1), -184662 (2). Copies may be requested free of charge from The Director, CCDC, 12 Union Road, Cambridge CB2 1EZ, England (E-mail: deposit@ccdc.cam.ac.uk),

9. (a) Woodward, R. B.; Hoffmann, R. The Conservation of Orbital Symmetry; Academic Press: New York, 1972; (b) Fleming, I. Frontier Orbitals and Organic Chemical Reactions; John Wiley and Sons: Chichester, 1978.

10. (a) Corey, E. J.; Cheng, X.-M. The Logic of Chemical Synthesis; John Wiley and Sons: New York, 1989; (b) Chanon, M.; Barone, R.; Baralotto, C.; Julliard, M.; Hendrickson, J. B. Synthesis 1998, 1559. 